\title{
NEVANLINNA-PICK INTERPOLATION ON SOBOLEV SPACE
}

\author{
JIM AGLER
}

(Communicated by John B. Conway)

\begin{abstract}
In this paper we shall prove an analog of the classical result of Nevanlinna and Pick concerning the bound of holomorphic functions on the unit disc that take prescribed values at prescribed points with the role that the classical Hardy space of analytic functions with square integrable power series plays in the modern operator-theoretic formulation of that result instead played by a Sobolev space of complex functions on the interval $[0,1]$ whose derivatives are in $L^{2}(0,1)$.
\end{abstract}

\section{INTRODUCTION}

Let $H^{2}$ denote the classical Hardy space of analytic functions defined on the open unit disc $\mathrm{D}$ and with square summable power series. $H^{2}$ is a Hilbert space when equipped with the inner product,

$$
\left\langle\sum_{n=0}^{\infty} a_{n} z^{n}, \sum_{n=0}^{\infty} b_{n} z^{n}\right\rangle=\sum_{n=0}^{\infty} a_{n} \bar{b}_{n} .
$$

Let $H^{\infty}$ denote the space of functions in $H^{2}$ that are bounded on D. $H^{\infty}$ is a Banach space when equipped with the norm,

$$
\|\phi\|_{\infty}=\sup \{\mid \phi(\gamma) \| \gamma \in \mathbf{D}\} .
$$

Suppose that $n$ points $z_{1}, \ldots, z_{n} \in \mathbf{D}$ are given and that it is desired to obtain a function $\phi \in H^{\infty}$ of smallest possible norm that assumes given values $w_{1}, \ldots, w_{n} \in \mathbf{C}$ at the points $z_{1}, \ldots, z_{n}$. The following well-known result which goes back to Pick [2] and Nevanlinna [1] effectively answers this question.

Theorem 0.1. Let $z_{1}, \ldots, z_{n} \in \mathbf{D}$ and let $w_{1}, \ldots, w_{n} \in \mathbf{C}$. There exists $\phi \in$ $H^{\infty}$ with $\|\phi\|_{\infty} \leq 1$ and $\phi\left(z_{i}\right)=w_{i}$ whenever $1 \leq i \leq n$ if and only if the $n \times n$ matrix $\left(1-\bar{w}_{i} w_{j} / 1-\bar{z}_{i} z_{j}\right)$ is positive definite.

Received by the editors December 29, 1986. The contents of this paper were presented at the CBMS regional conference in Lincoln, Nebraska, 1985.

1980 Mathematics Subject Classification (1985 Revision). Primary 47A20, 30C40, 46E39.

Work partially supported by NSF grants DMS84-09630 and DMS89-03235. 
In Theorem 0.1 to say that an $n \times n$ matrix $M=\left(m_{i j}\right)$ is positive definite $(M \geq 0)$ means that

$$
\sum_{i, j=1}^{n} m_{i j} c_{j} \bar{c}_{i} \geq 0
$$

for all $c_{1}, \ldots, c_{n} \in \mathbf{C}$.

Theorem 0.1 has been the object of attention of the operator theory community for many years and its influence on the literature since Sarason's profound paper [3] appeared, has been extraordinary. We shall not attempt to review this literature here, but rather content ourselves with the trivial observation that is the basic reason that Theorem 0.1 has such a rich operator-theoretic context. Define an operator $S$ on $H^{2}$ by

$$
S f(z)=z f(z), \quad f \in H^{2} .
$$

$S$, which is often referred to as the unilateral shift, is an isometry. A basic exercise is to show that $T$ is a bounded operator on $H^{2}$ which commutes with $S$ if and only if there exists a $\phi \in H^{\infty}$ ( $\phi$ is unique) such that

$$
T(f)(z)=\phi(z) f(z)
$$

for all $f \in H^{2}$ and $z \in \mathbf{D}$. Furthermore, if $T$ and $\phi$ are related as above then $\|T\|=\|\phi\|_{\infty}$. Thus, properly interpreted $H^{\infty}$ is the commutant of $S$, the operator multiplication by $z$ on $H^{2}$.

In this paper we shall prove a precise analog of Theorem 0.1 with $H^{2}$ replaced by a Sobolev space $H$, and $H^{\infty}$ replaced with the commutant of the operator "multiplication by $t$ " on $H$. More exactly, let $H$ denote the set of functions defined and absolutely continuous on the closed interval $[0,1]$, and whose derivatives are in $L^{2}(0,1) . H$ is a Hilbert space with inner product given by

$$
[f, g]=\int_{0}^{1} f \bar{g}+\int_{0}^{1} f^{\prime} \bar{g}^{\prime} .
$$

If we define an operator $M_{t}$ on $H$ by the formula,

$$
M_{t}(f)(t)=t f(t), \quad t \in[0,1]
$$

it is elementary to ascertain that the commutant of $M_{t}$ is the collection of all operators on $H$ that have the form,

$$
M_{\phi}(f)(t)=\phi(t) f(t)
$$

for some $\phi \in H$. Thus, the space that should play the role that $H^{\infty}$ plays in the classical Nevanlinna-Pick Theorem is now $H$, equipped with the norm defined by

$$
\|\phi\|_{\infty}=\sup \{\|\phi f\| \mid f \in H \text { and }\|f\|=1\} .
$$

The interpolation problem we wish to solve is now clear. Given $t_{1}, \ldots, t_{n} \in$ $[0,1]$ and $w_{1}, \ldots, w_{n} \in \mathbf{D}$ when is it the case that there exists a $\phi \in H$ with $\|\phi\|_{\infty} \leq 1$ and $\phi\left(t_{i}\right)=w_{i}$ ? 
The answer, which is given by the following theorem, involves the kernel function for $H$. One observes that if $s \in[0,1]$, then one has a Sobolev inequality of the type,

$$
|f(s)| \leq c\|f\|
$$

for all $f \in H$. Consequently, by the Riesz Representation Theorem there exists $k_{s} \in H$ such that

$$
f(s)=\left[f, k_{s}\right]
$$

for all $f \in H$. The two-variable function $k(s, t)$ defined by

$$
k(s, t)=k_{s}(t)=\left[k_{s}, k_{t}\right]
$$

is referred to as the kernel function for $H$. Note that if one duplicates the above procedure for $H^{2}$, then one obtains the Szego kernel defined by

$$
k(\lambda, z)=\frac{1}{1-\bar{\lambda} z}, \quad \lambda, z \in \mathbf{D},
$$

and that the condition for interpolation given in Theorem 0.1 is the assertion that

$$
\left(\left(1-\bar{w}_{i} w_{j}\right) k\left(z_{i}, z_{j}\right)\right) \text { is positive definite. }
$$

Theorem 0.2. Let $k$ denote the kernel function for the space $H$. Let $t_{1}, \ldots, t_{n} \in$ $[0,1]$ and let $w_{1}, \ldots, w_{n} \in \mathbf{C}$. There exists $\phi \in H$ with $\|\phi\|_{\infty} \leq 1$ and $\phi\left(t_{i}\right)=$ $w_{i}$ whenever $1 \leq i \leq n$ if and only if the $n \times n$ matrix $\left(\left(1-\bar{w}_{i} w_{j}\right) k\left(t_{i}, t_{j}\right)\right)$ is positive definite.

The remainder of this paper is divided into two sections. In $\S 1$ we state the basic facts about $H$ that are required for the proof of Theorem 0.2. For the convenience of the reader we give proofs of those facts which appear in $\S 1$ which are not immediately apparent from the definitions, even though proofs might appear elsewhere or be obvious to some readers. In $\S 2$ we present a proof of Theorem 0.2.

We are deeply indebted to Richard Froese who supplied the idea of the proof of the claim used in the proof of Theorem 1.5.

\section{Some basic Facts ABout $H$}

Let $H$ denote the space of absolutely continuous complex-valued functions defined on $[0,1]$ whose derivatives are in $L^{2}(0,1) . H$ is a Hilbert space when equipped with inner product given by

$$
[f, g]=\int_{0}^{1} f(t) \overline{g(t)} d t+\int_{0}^{1} f^{\prime}(t) \overline{g^{\prime}(t)} d t .
$$

In this section we shall adhere to the notations

$$
\langle f, g\rangle=\int_{0}^{1} f(t) \overline{g(t)} d t
$$

and

$$
\|f\|^{2}=[f, f] .
$$


If $\psi$ is a function defined on $[0,1]$ and we attempt to define a transformation $M_{\psi}$ of $H$ by

$$
M_{\psi} f=\psi f,
$$

then it is easy to ascertain that $M_{\psi}$ maps $H$ into $H$ if and only if $M_{\psi}$ is a bounded operator if and only if $\psi \in H$. In the introduction of the paper we used the notation $\|\psi\|_{\infty}$ for the norm of $M_{\psi}$ as an operator on $H$ (to create the analogy with $H^{\infty}$ ). In the sequel we shall eschew this notation and write

$$
\left\|M_{\psi}\right\| \quad \text { for } \sup \{\|\psi f\| \mid f \in H,\|f\|=1\} .
$$

Another simple fact about $H$ is that point evaluations are continuous. Thus, if $s \in[0,1]$ then there exists a constant $c_{s}$ such that

$$
|f(s)| \leq c_{s}\|f\|
$$

for all $f \in H$. By the Riesz Representation Theorem it follows that if $s \in$ $[0,1]$, then there exists $k_{s} \in H$ such that

$$
f(s)=\left[f, k_{s}\right]
$$

for all $f \in H$. Direct computation reveals that

where

$$
k_{s}(t)=\left\{\begin{array}{ll}
a_{s} e^{t}+b_{t} e^{-t}, & t \leq s, \\
c_{s} e^{t}+d_{s} e^{-t}, & t \geq s,
\end{array} \quad 0 \leq s, t \leq 1,\right.
$$

$$
\begin{aligned}
& a_{s}=b_{s}=\frac{e^{s}+e^{2} e^{-s}}{2\left(e^{2}-1\right)}, \\
& c_{s}=\frac{e^{s}+e^{-s}}{e^{2}-1,}
\end{aligned}
$$

and

$$
d_{s}=e^{2} c_{s}
$$

In particular, we obtain the following important fact.

Lemma 1.1. $k_{s}(t) \neq 0$ for all $s, t \in[0,1]$.

The following lemma will follow quickly from Lemma 1.1. Note that if $\phi \in H$ and $s \in[0,1]$, then $M_{\phi}^{*} k_{s}=\overline{\phi(s)} k_{s}$.

Lemma 1.2. If $\phi \in H$ and $\phi$ is not constant, then

$$
\left\|M_{\phi}\right\|>\max \{\mid \phi(t) \| t \in[0,1]\} \text {. }
$$

Proof. If not, then there exists $t_{0} \in[0,1]$ such that $\left\|M_{\phi}\right\|=\left|\phi\left(t_{0}\right)\right|$. Thus,

$$
\left\langle M_{\phi} M_{\phi}^{*} k_{t_{0}}, k_{t_{0}}\right\rangle=\left|\phi\left(t_{0}\right)\right|^{2}\left\|k_{t_{0}}\right\|^{2}=\left\|M_{\phi} M_{\phi}^{*}\right\|\left\|k_{t_{0}}\right\|^{2}
$$

and we see that $M_{\phi}^{*} k_{t_{0}}=\overline{\phi\left(t_{0}\right)} k_{t_{0}}$ is an eigenfunction for $M_{\phi}$. Hence

$$
\phi k_{t_{0}}=\phi\left(t_{0}\right) k_{t_{0}}
$$

and Lemma 1.1 implies that $\phi$ is constant. This establishes Lemma 1.2. 
The following lemma will allow us to prove that multipliers of $H$ attain their norms on nonvanishing vectors.

Lemma 1.3. If $\phi \in H$ is not constant, and $\rho=\left\|M_{\phi}\right\|$, then there exists $a$ nonvanishing $\psi \in H$ and $\nu \in L^{1}(0,1)$ such that

$$
\left[\left(\rho^{2}-M_{\phi}^{*} M_{\phi}\right) f, f\right]=[\psi f, \psi f]+\langle\nu f, f\rangle
$$

for all $f \in H$.

Proof. Define $\psi$ by

$$
\psi(t)=e^{i w(t)}\left(\rho^{2}-|\phi(t)|^{2}\right)^{1 / 2},
$$

where $w^{\prime}(t)=\operatorname{Im}\left(\phi \bar{\phi}^{\prime}\right) /\left(\rho^{2}-|\phi|^{2}\right)$. By Lemma 1.2, there exists $\delta>0$ such that $\rho^{2}-|\phi(t)|^{2} \geq \delta$ for all $t \in[0,1]$ and thus, $\psi$ is well defined, $\psi \in H$ and $\psi$ does not vanish on $[0,1]$. Also direct calculation shows that

$$
|\psi|^{2}=\rho^{2}-|\phi|^{2}
$$

and

$$
\psi \bar{\psi}^{\prime}=-\phi \bar{\phi}^{\prime}
$$

Calculating, using these last identities shows that if $f \in H$, then

$$
\left[\left(\rho^{2}-M_{\phi}^{*} M_{\phi}\right) f, f\right]=[\psi f, \psi f]+\langle\nu f, f\rangle,
$$

where

$$
\nu=-\left|\phi^{\prime}\right|^{2}-\left|\psi^{\prime}\right|^{2}
$$

This completes the proof of Lemma 1.3.

Lemma 1.4. If $\nu \in L^{1}$ and $K \in L(H)$ is defined by

$$
[K f, g]=\langle\nu f, g\rangle, \quad f, g \in H,
$$

then $K$ is compact.

Theorem 1.5. Let $\phi \in H$ and assume $\phi$, is not constant. The norm of $M_{\phi}$ is attained, i.e. there exists a vector $f_{0} \in H$ such that $\left\|f_{0}\right\| \neq 0$ and $\left\|\phi f_{0}\right\|=$ $\left\|M_{\phi}\right\|\left\|f_{0}\right\| . \quad f_{0}$ is unique up to a scalar factor (i.e. if $g \in H, g \neq 0$ and $\left\|M_{\phi} g\right\|=\left\|M_{\phi}\right\|\|g\|$, then $f_{0}$ and $g$ are linearly dependent). There exists a $\delta>0$ such that if $h \perp f_{0}$, then

$$
\|\phi h\| \leq\left(\left\|M_{\phi}\right\|-\delta\right)\|h\| .
$$

Finally, $f_{0}$ does not vanish on $[0,1]$.

Proof. Let $\rho=\left\|M_{\phi}\right\|$. By Lemma 1.3, there exist $\psi \in H$ and $\nu \in L^{1}$ such that $\psi$ does not vanish on $[0,1]$ and

$$
\left[\left(\rho^{2}-M_{\phi}^{*} M_{\phi}\right) f, f\right]=[\psi f, \psi f]+\langle\nu f, f\rangle
$$

for all $f \in H$. 
Define an operator $K$ on $H$ by requiring that

$$
[K f, g]=\langle\nu f, g\rangle \text {. }
$$

By Lemma 1.4, $K$ is compact. Combining (1.7) and (1.8) we see that,

$$
\rho^{2}-M_{\phi}^{*} M_{\phi}=M_{\psi}^{*} M_{\psi}+K .
$$

Since $\psi$ does not vanish on $[0,1], M_{\psi}$ is boundedly invertible. Choose a sequence $f_{n} \in H$ such that $\left\|f_{n}\right\|=1$ and $\left\|\phi f_{n}\right\| \rightarrow \rho$. Passing to a subsequence if necessary we may assume that $f_{n} \rightarrow f_{0}$ weakly in $H$. Thus, $K f_{n} \rightarrow K f_{0}$ and $\left(\rho^{2}-M_{\phi}^{*} M_{\phi}\right) f_{n} \rightarrow 0$ so that from (1.9) we see that $M_{\psi}^{*} M_{\psi} f_{n} \rightarrow-K f_{0}$. Consequently, $f_{n} \rightarrow-\left(M_{\psi}^{*} M_{\psi}\right)^{-1} K f_{0},\left\|f_{0}\right\|=1$, and $\left[\left(\rho_{1}^{2}-M_{\phi}^{*} M_{\phi}\right) f_{0}, f_{0}\right]=0$ i.e. $M_{\phi}$ attains its norm.

Let $N=\{f \mid\|\phi f\|=\rho\|f\|\}$. Cauchy's inequality shows that $N$ is a subspace of $H$. The argument in the preceding paragraph shows that there exists a $\delta>0$ such that $\|\phi h\| \leq(\rho-\delta)\|h\|$ whenever $h \perp N$. Thus, the proof of Theorem 1.5 will be complete once we have shown that $\operatorname{dim} N=1$ and $f \in N, f \neq 0$ imply $f$ does not vanish on $[0,1]$. In fact, it is enough to show that if $f \in N$ and $f \neq 0$, then $f$ does not vanish on $[0,1]$ (if $\operatorname{dim} N>1$, then $N$ must contain a nonzero vector with a 0 on the interval). This we now do.

Let $w=\nu /|\psi|^{2}+1$ and define a sesquilinear form on $H$ by

$$
(f, g)=\int f^{\prime} \bar{g}^{\prime}+\int w f \bar{g} .
$$

Evidently, since $\left.(f, g)=\left[\left(\rho^{2}-M_{\varphi}^{*} M_{\varphi}\right) f / \psi\right), g / \psi\right]$,

$$
(f, f) \geq 0 \quad \text { whenever } f \in H,
$$

and

$$
\left(\psi f_{0}, \psi f_{0}\right)=0 \quad \text { if } f_{0} \in N .
$$

Claim. If $h \in H$ and $(h, h)=0$, then $h^{\prime}$ is absolutely continuous, $h^{\prime \prime} \in$ $L^{1}(0,1), h^{\prime}(0)=h^{\prime}(1)=0$ and $-h^{\prime \prime}+w h=0$ a.e.

Before establishing the claim we first show how it establishes that if $f \in N$ and $f \neq 0$ then $f$ does not vanish on $[0,1]$. We argue by contradiction. Thus, suppose that $f \in N, f \neq 0$, and $f(c)=0$. Define $h=\psi f$. Thus, $h \in H$ and by (1.11), $(h, h)=0$. By the claim, $h \in L^{1}, h^{\prime}(0)=h^{\prime}(1)=0$, and $-h^{\prime \prime}+w h=0$ a.e. Thus $c \neq 0,1$ (otherwise $h \equiv 0$ ). Define $h_{-}$and $h_{+}$by

$$
h_{+}(t)= \begin{cases}0 & \text { if } t \leq c, \\ h(t) & \text { if } t \geq c\end{cases}
$$

and

$$
h_{-}(t)= \begin{cases}h(t) & \text { if } t \leq c, \\ 0 & \text { if } t \geq c .\end{cases}
$$


Apparently, $h_{ \pm}(t) \in H$ and $\left(h_{ \pm}, h_{ \pm}\right)=0$ so that by the claim, $h_{ \pm}^{\prime \prime}+w h_{ \pm}=0$. This last absurdity establishes that the assumption $f(c)=0$ was bogus and finishes the proof of Theorem 1.5.

To establish the claim assume that $h \in H$ and $(h, h)=0$. By $(1.10)(\cdot, \cdot)$ satisfies Cauchy's inequality and thus

$$
\int_{0}^{1} h^{\prime} \bar{\phi}^{\prime}+\int_{0}^{t} w h \bar{\phi}=0
$$

whenever $\phi \in C^{\infty}([0,1])$. Letting $\phi(t)=\phi(0)+\int_{0}^{t} \phi^{\prime}(s) d s$ we see that

$$
\begin{aligned}
0= & \int_{0}^{1} h^{\prime} \bar{\phi}^{\prime}+\int_{0}^{1} w(h) h(t)\left(\overline{\phi(0)}+\int_{0}^{1} \overline{\phi^{\prime}(s)} d s\right) d t \\
= & \overline{\phi(0)} \int_{0}^{1} w(t) h(t) d t+\int_{0}^{1} h^{\prime} \bar{\phi}^{\prime}+\int_{0}^{1} \int_{0}^{1} w(t) h(t) \chi_{[0, t]}(s) \overline{\phi^{\prime}(s)} d s d t \\
& +\int_{0}^{1} \int_{0}^{1} w(t) h(t) \chi_{[0, t]}(s) \overline{\phi^{\prime}(s)} d s d t \\
= & \overline{\phi(0)} \int_{0}^{1} w(t) h(t) d t+\int_{0}^{1}\left(h^{\prime}(t)+\int_{t}^{1} w(s) h(s) d s\right) \overline{\phi^{\prime}(t) d t}
\end{aligned}
$$

whenever $\phi \in C^{\infty}([0,1])$. Thus, $\int_{0}^{1} w h=0$ and

$$
h^{\prime}(t)+\int_{t}^{1} w(s) h(s) d s=0 \quad \text { for a.e. } t .
$$

It follows that $h^{\prime}$ is absolutely continuous and that

$$
h^{\prime \prime}-w h=0 \quad \text { for a.e. } t \text {. }
$$

Since $w \in L^{1}$ we have that $h^{\prime \prime} \in L^{1}$. From (1.12) we see that $h^{\prime}(1)=0$ and also (since $\int_{0}^{1} w h=0$ ) that $h^{\prime}(0)=0$. This concludes the proof of the claim.

\section{THE PROOF}

In this section $t_{1}, \ldots, t_{n} \in[0,1]$ and $w_{1}, \ldots, w_{n} \in \mathbf{C}$ will be fixed. We shall assume not all of the $w_{i}$ 's are equal. Let

$$
I=\left\{\phi \in H \mid \phi\left(t_{i}\right)=0 \text { whenever } 1 \leq i \leq n\right\},
$$

and

$$
\rho=\inf \left\{\left\|M_{\phi}\right\| \mid \phi\left(t_{i}\right)=w_{i} \text { whenever } 1 \leq i \leq n\right\} .
$$

Our first observation is that $\rho$, which is defined as an infimum, is actually attained.

Lemma 2.1. There exists $\psi \in H$ such that $\psi\left(t_{i}\right)=w_{i}$ and $\left\|M_{\psi}\right\|=\rho$.

Proof. Choose $\phi_{j}$ such that $\phi_{j}\left(t_{i}\right)=w_{i}$ for all $j \geq 1$ and $1 \leq i \leq n$ and such that $\left\|M_{\phi_{j}}\right\| \rightarrow \rho$ as $j \rightarrow \infty$. Replacing $\left\{\phi_{j}\right\}$ with a subsequence if necessary 
we may assume that there exists a $T \in L(H)$ such that $M_{\phi_{j}} \rightarrow T$ in the $w^{*}$ topology of $L(H)$. In particular,

$$
\left\langle T k_{s}, k_{t}\right\rangle=\lim _{j \rightarrow \infty}\left\langle M_{\phi_{j}} k_{s}, k_{t}\right\rangle=\lim _{j \rightarrow \infty} \phi_{j}(t) k_{s}(t)
$$

whenever $s, t \in[0,1]$. Thus, by Lemma 1.1 there exists a function $\psi$ defined on $[0,1]$ such that

$$
\lim _{j \rightarrow \infty} \phi_{j}(t)=\psi(t)
$$

for all $t \in[0,1]$. In particular, $\psi\left(t_{i}\right)=w_{i}$ whenever $1 \leq i \leq n$, and from (2.2) we see that

$$
\left(T k_{s}\right)(t)=\left\langle T k_{s}, k_{t}\right\rangle=\psi(t) k_{s}(t)=\left(\psi k_{s}\right)(t) .
$$

Thus, by Lemma 1.1,

$$
\psi=\frac{T k_{s}}{k_{s}} \in H,
$$

and $T=M_{\psi}$. Finally, since

$$
\left\|M_{\psi}\right\|=\|T\| \leq \varlimsup_{j \rightarrow \infty}\left\|M_{\phi_{j}}\right\|=\rho
$$

we see that in fact $\left\|M_{\psi}\right\|=\rho$ which concludes the proof of Lemma 2.1.

Now fix $\psi_{0} \in H$ such that $\psi_{0}\left(t_{i}\right)=w_{i}$ whenever $1 \leq i \leq n$ and $\left\|M_{\psi_{0}}\right\|=\rho$. Since not all of the $w_{i}$ 's are equal, $\psi_{0}$ is not constant, and thus by Theorem 1.5 there exists a vector $f_{0}$ such that

$$
\left\|f_{0}\right\|=1 \text { and }\left\|\psi_{0} f_{0}\right\|=\rho .
$$

Claim. $\left[\omega f_{0}, \psi_{0} f_{0}\right]=0$ whenever $\omega \in I$.

The proof of the claim will be by contradiction and will use the following lemma.

Lemma 2.4. Let $A \in L(H), A=A^{*}$, and suppose that $f_{0} \in H . A \geq 0$ if and only if

(i) $\langle A h, h\rangle \geq 0$ for all $h \perp f_{0}$,

(ii) $\left\langle A f_{0}, f_{0}\right\rangle \geq 0$, and

(iii) $\left|\left\langle A h, f_{0}\right\rangle\right|^{2} \leq\left\langle A f_{0}, f_{0}\right\rangle\langle A h, h\rangle$ for all $h \perp f_{0}$.

To prove Lemma 2.4 complete the square in $\lambda$ on the expansion of

$$
\left\langle\left(\lambda f_{0}+h\right)<\left(\lambda f_{0}+h\right)\right\rangle .
$$

We now prove the claim. Define for $t \geq 0$,

$$
A_{t}=\rho^{2}-t^{2}-M_{\psi_{0}+t \omega}^{*} M_{\psi_{0}+t \omega} .
$$

We shall show that if the claim is false, then there exists $\omega \in I$ such that $A_{t} \geq 0$ for all sufficiently small $t>0$. This would contradict the definition of $\rho$ and thus establish the claim. 
Assume the claim is false. Thus there exists $\omega \in I$ with $\left[\omega f_{0}, \psi_{0} f_{0}\right] \neq 0$. Scaling $\omega$ if necessary, we see that there exists $\omega \in I$ and a constant $c>0$ such that

$$
\left[\omega f_{0}, \psi_{0} f_{0}\right]=-c<0 .
$$

We now use Lemma 2.4 to show that $A_{t} \geq 0$ for all sufficiently small $t>0$. We need to verify conditions (i), (ii), and (iii) for small $t$.

(i). Since $A_{0}=\rho^{2}-M_{\psi_{0}}^{*} M_{\psi_{0}}$, Theorem 1.5 implies that there exists $\delta>0$ such that $\left\langle A_{0} h, h\right\rangle \geq \delta\|h\|^{2}$ whenever $h \perp f_{0}$. By the continuity of $A_{i}$ there exists $\delta_{1}$ such that for sufficiently small $t$,

$$
\left[A_{t} h, h\right] \geq \delta_{1}\|h\|^{2} \quad \text { whenever } h \perp f_{0} .
$$

(ii). From (2.3) we see that

$$
\begin{aligned}
{\left[A_{t} f_{0}, f_{0}\right]=} & {\left[A_{0} f, f_{0}\right]-t^{2}\left[f_{0}, f_{0}\right] } \\
& -t\left(\left[\omega f_{0}, \psi_{0} f_{0}\right]+\left[\psi_{0} f_{0}, \omega f_{0}\right]\right)-t^{2}\left[\omega f_{0}, \omega f_{0}\right] \\
= & 2 c t-t^{2}\left(1+\left\|\omega f_{0}\right\|^{2}\right) .
\end{aligned}
$$

Hence $\left[A_{t} f_{0}, f_{0}\right] \geq 0$ if $t$ is sufficiently small.

(iii). From Cauchy's inequality and the proof of (i) we have

$$
\left|\left[A_{t} h, f_{0}\right]\right|^{2} \leq\|h\|^{2}\left\|A_{t} f_{0}\right\|^{2} \leq\left[A_{t} h, h\right]\left(\frac{1}{\delta_{1}}\left\|A_{t} f_{0}\right\|^{2}\right) .
$$

From (2.3), we have that

$$
A_{t} f_{0}=-t^{2} f_{0}-t\left(M_{\omega}^{*} M_{\psi_{0}}+M_{\psi_{0}}^{*} M_{\omega}\right) f_{0}-t^{2} M_{\omega}^{*} M_{\omega} f_{0} .
$$

Thus, $\left\|A_{t} f_{0}\right\|^{2}=\mathrm{O}\left(t^{2}\right)$ but from (ii) $\left[A_{t} f_{0}, f_{0}\right]=2 c t+\mathrm{O}\left(t^{2}\right)$. Consequently,

$$
\left|\left[A_{t} h, f_{0}\right]\right|^{2} \leq\left[A_{t} h, h\right]\left[A_{t} f_{0}, f_{0}\right]
$$

for sufficiently small $t$.

We have shown that conditions (i), (ii), and (iii) in Lemma 2.4 hold and thus that $A_{t} \geq 0$ when $t$ is sufficiently small. This contradicts the definition of $\rho$ and we have established the claim.

The following proposition describes the key qualitative information that occurs when the optimum is realized by $\psi_{0}$ and $f_{0}$ as above.

Proposition 2.6. Let $t_{1}, \ldots, t_{n} \in[0,1]$ and $w_{1}, \ldots, w_{n} \in \mathbf{C}$ be given. If

$$
\begin{aligned}
\left\|M_{\psi_{0}}\right\| & =\inf \left\{\left\|M_{\phi}\right\| \mid \phi\left(t_{i}\right)=w_{i} \text { whenever } 1 \leq i \leq n\right\}, \\
\psi_{0}\left(t_{i}\right) & =w_{i} \quad \text { whenever } 1 \leq i \leq n,
\end{aligned}
$$

and

$$
\left\|M_{\psi_{0}} f_{0}\right\|=\left\|M_{\psi_{0}}\right\|\left\|f_{0}\right\|
$$

where $f_{0} \neq 0$ and $\psi_{0}$ is not constant, then $f_{0}$ and $\psi_{0} f_{0}$ are orthogonal to $I$. 
Proof. From the claim we have that

$$
\left[\omega f_{0}, \psi_{0} f_{0}\right]=0
$$

whenever $\omega \in I$. Since $\psi_{0}$ is not constant by Theorem 1.5 we know that $f_{0}$ does not vanish on $[0,1]$. Hence $\left\{\omega f_{0} \mid \omega \in I\right\}=I$. Thus $\psi_{0} f_{0} \perp I$. Since

$$
\left[\left(\rho^{2}-M_{\psi_{0}}^{*} M_{\psi_{0}}\right) f_{0}, f_{0}\right]=0
$$

and

we have that

$$
\rho^{2}-M_{\psi_{0}}^{*} M_{\psi_{0}} \geq 0
$$

$$
M_{\psi_{0}}^{*}\left(\psi_{0} f\right)=\rho^{2} f_{0} .
$$

Since $\psi_{0} f \perp I$ and $H \ominus I$ is invariant for $M_{\psi_{0}}^{*}$ we deduce that $f_{0} \perp I$. This concludes the proof of Proposition 2.6.

The following theorem is only trivially more general than Theorem 0.2 from the introduction of the paper.

Theorem 2.7. Let $k$ denote the kernel function for the space $H$. Let $t_{1}, \ldots, t_{n} \in$ $[0,1]$ and let $w_{1}, \ldots, w_{n} \in \mathbf{C}$. There exists $\phi \in H$ with $\left\|M_{\phi}\right\| \leq \rho$ and $\phi\left(z_{i}\right)=w_{i}$ if and only if

$$
\left(\left(\rho^{2}-\bar{w}_{i} w_{j}\right) k\left(t_{i}, t_{j}\right)\right) \geq 0 .
$$

Proof. First assume that $\phi \in H$ with $\left\|M_{\phi}\right\| \leq \rho$ and $\phi\left(z_{i}\right)=w_{i}$. In particular, $\| M_{\phi}^{*} \mid\left(H \ominus I \| \leq \rho\right.$. Thus, for arbitrary constants $c_{1}, \ldots, c_{n} \in \mathbf{C}$ we have

$$
\left\|M_{\phi}^{*}\left(\sum c_{i} k_{t_{i}}\right)\right\|^{2} \leq \rho^{2}\left\|\sum c_{i} k_{t_{i}}\right\|^{2} .
$$

Unraveling this last inequality using the fact that $M_{\phi}^{*} k_{t_{i}}=\bar{w}_{i} k_{t_{i}}$ yields (2.8).

Now assume (2.8) holds. If $w_{i}=w$ for all $i$, then $|w|^{2} \leq \rho^{2}$ and $\phi \equiv w$ does the job. Thus, we may assume not all of the $w_{i}$ 's are equal. Define

$$
\rho_{0}=\inf \left(\left\|M_{\phi}\right\| \mid \phi\left(t_{i}\right)=w_{i} \text { whenever } 1 \leq i \leq n\right\} .
$$

Evidently, we need to show that $\rho_{0} \leq \rho$. Using Lemma 2.1 choose $\psi_{0}$ such that $\psi_{0}\left(t_{i}\right)=w_{i}$ and $\left\|M_{\psi_{0}}\right\|=\rho$. By Theorem 1.5 choose $f_{0}$ so that $\left\|f_{0}\right\|=1$ and $\left\|\psi_{0} f_{0}\right\|=\rho_{0}$. By Proposition 2.6, $\psi_{0} f_{0} \perp I$ and $M_{\psi_{0}}^{*}\left(\psi_{0} f_{0}\right)=\rho_{0}^{2} f_{0}$. It follows that

$$
\left(\left(\rho_{0}^{2}-\bar{w}_{i} w_{j}\right) k\left(t_{i}, t_{j}\right)\right) \geq 0
$$

and furthermore that if $\psi_{0} f_{0}=\sum \bar{c}_{i} k_{t_{i}}$, then

$$
\sum_{i j}\left(\rho_{0}^{2}-\bar{w}_{i} w_{j}\right) k\left(t_{i}, t_{j}\right) c_{j} \bar{c}_{i}=0 .
$$

From (2.8), (2.9), (2.10), and the fact that $\left(k\left(t_{i}, t_{j}\right)\right)$ is nonsingular and positive definite we conclude that $\rho_{0} \leq \rho$. This completes the proof of Theorem 2.7. 


\section{REFERENCES}

1. R. Nevanlinna, Über Beschränkte Funktionen die in gegebene Punkten vorgeschriebene Werte annehmen, Ann. Acad. Sci. Fenn. Ser. A 13 (1), (1919), 1-71.

2. G. Pick, Über die Beschränkungen analytischer Funktionen, welche durch vorgegebene Funktionswerte bewirkt werden, Math. Ann. 77 (1916), 7-23.

3. D. Sarason, Generalized interpolation in $H^{\infty}$, Trans. Amer. Math. Soc. 127 (1967), 179-203.

Department of Mathematics, University of California, San Diego, la Jolla, CALIFORNIA 92093 